\title{
Photoluminescence study of the self-organized InAs/GaAs quantum dots grown by gas source molecular beam epitaxy
}

\author{
Ming-Chin Chen and Hao-Hsiung Lin \\ Room 419,Department of Electrical Engineering, \\ National Taiwan University, Taipei, Taiwan, R. O. C.
}

\begin{abstract}
Self-organized InAs quantum dots (QDs) grown on both (100) exact and (100) misoriented $7^{\circ}$ toward (110) GaAs substrates using gas source molecular beam epitaxy (GSMBE) with V/IIl ratio ranging from 1.1 to 20 have been studied by photoluminescence (PL) measurements from 8.5 to $300 \mathrm{~K}$. The QD structures grown on the misoriented substrates show a better uniformity than those grown on the exact substrates at the same growth conditions. Effects of $\mathrm{AsH}_{3}$ flow rate on the PL intensity, peak energy and linewidth for QDs grown on both types of substrates are presented. Basically, higher $\mathrm{AsH}_{3}$ flow rate gives higher $\mathrm{PL}$ intensity at $8.5 \mathrm{~K}$. At room temperature $(300 \mathrm{~K})$, on the contrary, lower $\mathrm{AsH}_{3}$ flow rate results in higher PL intensity. The study of thermal quenching energy reveals that the larger the dot size the better the PL intensity at $300 \mathrm{~K}$.
\end{abstract}

\section{Introduction}

Recently, strain-induced In(Ga)As/GaAs QDs have been intensely studied because these QDs show an efficient carrier confinement which can be used for the realization of novel optoelectronic devices like quantum dot lasers. Such QD lasers with low threshold current density and high characteristics temperature $\left(T_{0}\right)$ have been demonstrated [1]. For practical applications in optoelectronic devices, QD structures with high density and good uniformity are necessary to achieve the desired active volume. There are several groups $[2,3,4]$ making great efforts on the conditions during the growth to improve the optical properties for QDs. The growth conditions include substrate temperature, V/III ratio, InAs growth rate and substrate orientation. Recent study on the effect of substrate orientation [3] shows that the QDs grown on (711)B substrate have better quantum efficiency than those on (511)B and (100) substrates. It implies that a slight misorientation for (100) substrate may improve the uniformity of the QDs. In this study, the QD structures are grown by using GSMBE at various V/III ratio

Table 1. Substrate orientation, $\mathrm{AsH}_{3}$ flow rate, $\mathrm{V} / \mathrm{III}$ ratio, thermal quenching energy and activation energy for all QDs samples.

\begin{tabular}{lccccc}
\hline Sample & Substrate orientation & $\begin{array}{c}\mathrm{AsH}_{3} \text { flow rate } \\
\text { (SCCM) }\end{array}$ & V/III ratio & $\begin{array}{c}\text { Thermal quenching } \\
\text { energy (meV) }\end{array}$ & $\begin{array}{c}\text { Activation energy } \\
\text { (meV) }\end{array}$ \\
\hline $\mathrm{C} 490 \mathrm{~A}$ & $(100)$ exact & 0.08 & 1.1 & 14 & 108 \\
\hline $\mathrm{C} 491 \mathrm{~A}$ & $(100)$ exact & 0.15 & 2 & 16 & 91 \\
\hline $\mathrm{C} 492 \mathrm{~A}$ & $(100)$ exact & 0.37 & 5 & 15 & 126 \\
\hline $\mathrm{C} 493 \mathrm{~A}$ & $(100)$ exact & 0.74 & 10 & 13 & 156 \\
\hline $\mathrm{C} 494 \mathrm{~A}$ & $(100)$ exact & 1.50 & 20 & 16 & 96 \\
\hline $\mathrm{C} 490 \mathrm{~B}$ & misoriented & 0.08 & 1.1 & 14 & 41 \\
\hline $\mathrm{C} 491 \mathrm{~B}$ & misoriented & 0.15 & 2 & 15 & 108 \\
\hline $\mathrm{C} 492 \mathrm{~B}$ & misoriented & 0.37 & 5 & 13 & 130 \\
\hline $\mathrm{C} 493 \mathrm{~B}$ & misoriented & 0.74 & 10 & 12 & 103 \\
\hline $\mathrm{C} 494 \mathrm{~B}$ & misoriented & 1.50 & 20 & 13 & \\
\hline \hline
\end{tabular}


during the growth of InAs layer. Exact and misoriented (100) GaAs substrates are also used to study the orientation effect.

\section{Experiments}

Ten QD samples were grown by using a VG V80H GSMBE system in this study. The growth procedure included a $200 \mathrm{~nm}$ thick buffer layer grown at $620^{\circ} \mathrm{C}$, an InAs layer deposited with a nominal thickness of 3 monolayers at $490^{\circ} \mathrm{C}$, a $10 \mathrm{~nm}$ GaAs layer grown at $490^{\circ} \mathrm{C}$ to avoid the In segregation, and a $40 \mathrm{~nm} \mathrm{GaAs}$ capping layer grown at $620^{\circ} \mathrm{C}$. The growth rate was $0.09 \mathrm{ML} / \mathrm{s}$ and the V/III ratio during the growth of InAs layer was changed from 1.1 to 20 for various samples. For comparison, exact (100) (samples C490A-C494A) and (100) with $7^{\circ}$ off-cut to (110) (samples C490B-C494B) semiinsulating GaAs substrates were used. In order to have the same growth conditions, they were mounted side by side on the same substrate holder. The $\mathrm{AsH}_{3}$ flow rates and V/III ratio of these samples were listed in Table 1. After growth, PL measurements were performed from 8.5 to $300 \mathrm{~K}$ to probe the quality of the QD structures.

\section{Results and Discussions}

Fig.1(a), (b), and (c) show the $8.5 \mathrm{~K} \mathrm{PL}$ peak energy, intensity and full width at half-maximum (FWHM) as a function of $\mathrm{AsH}_{3}$ flow rate, respectively. From Fig.1(a), we can see that QDs grown on the vicinal plane have higher peak energy, which indicates they are with smaller size. Takayoshi et. al. [5] reported a thicker critical thickness for InAs grown on (111) GaAs substrate. From their finding, InAs grown on misoriented (100) GaAs substrates is also expected to have thicker critical thickness as well. The smaller QD size for these misoriented samples resulted from their thicker wetting layer.

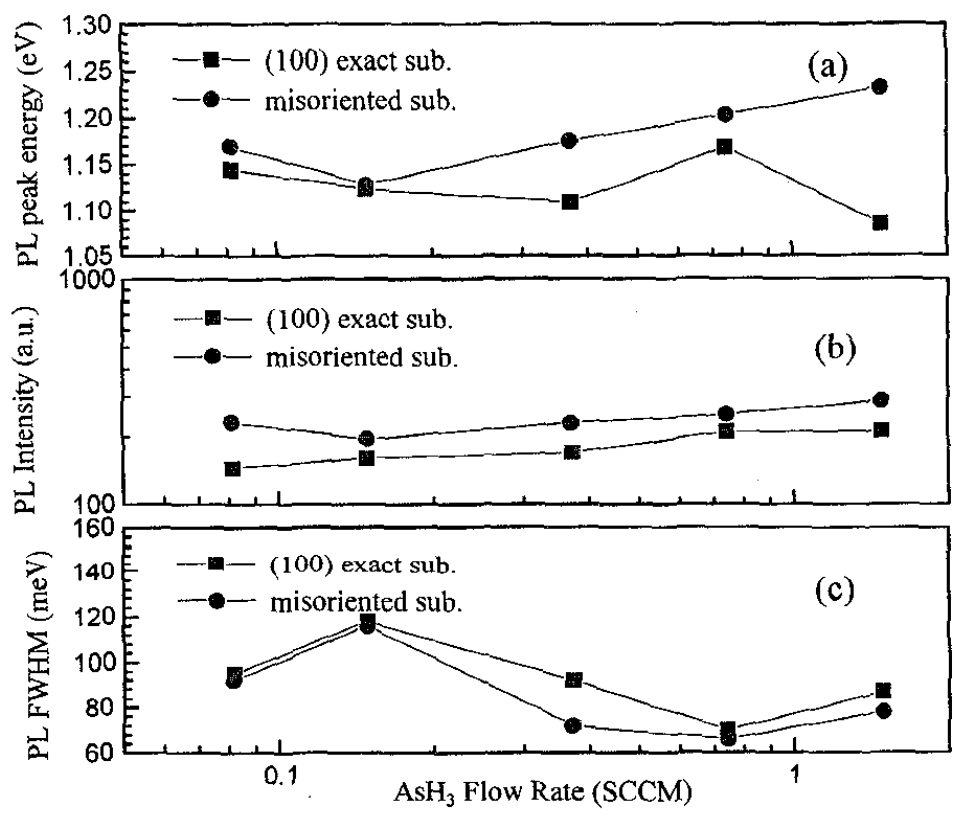

Fig.1. Spectra of the $8.5 \mathrm{~K}$ PL peak energy (a), intensity (b) and FWHM as a function of $\mathrm{AsH}_{3}$ flow rate. 

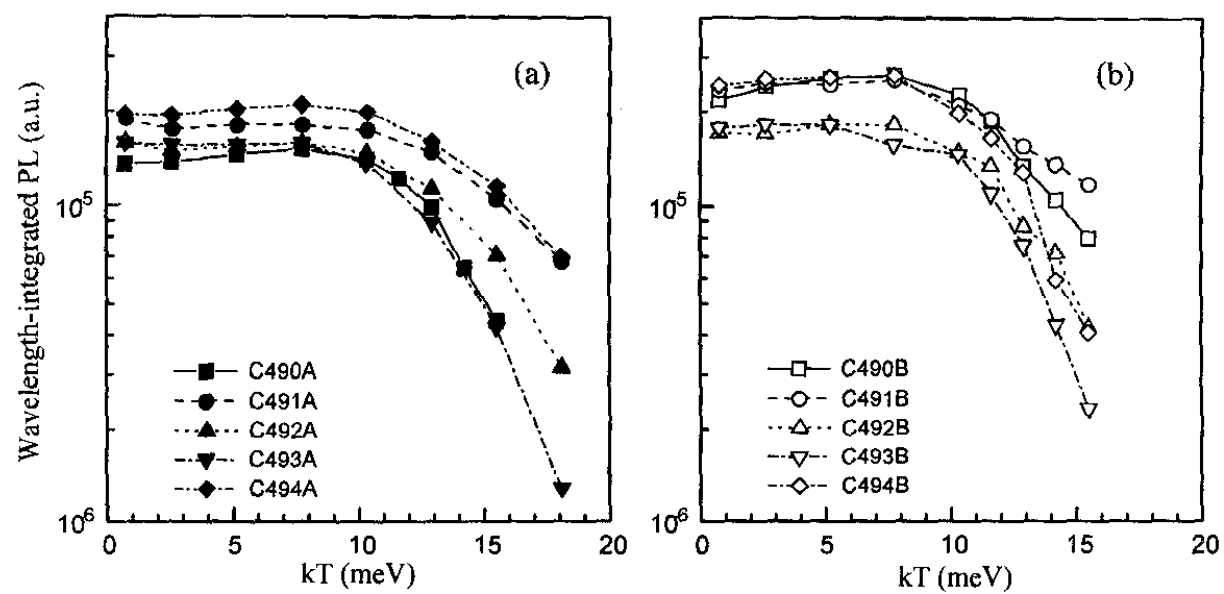

Fig. 2. Wavelength-integrated PL intensity as a function of thermal energy, kT, for QDs grown on the exact (a) and misoriented (b) substrates.

The PL peak energy of QDs grown on the vicinal surface increases with the increase of the V/III ratio. This phenomena is not found for the QDs grown on the exact substrates. A monotonously red-shift of PL peak, which implies the QD size increasing, with decreasing V/III ratio is shown in Fig. 1(a). It may be attributed to the increase of the In adatom migration length with decreasing $\mathrm{As}_{2}$ pressure under As-stabilized circumstance. There is an abnormal increasing of the PL peak energy when V/III ratio reduced to 1.1. In this condition, the growth circumstance of InAs may change from As-stabilized to In-stabilized, and the critical thickness of InAs is thus greatly enhanced [6]. The increase of the wetting layer thickness will result in the small dot size and the high PL peak energy. As shown in Fig. 1(b), PL intensity is enhanced when $\mathrm{AsH}_{3}$ flow rate increases. This trend is for QDs grown on both types of substrates. The increase of $\mathrm{AsH}_{3}$ flow rate will limit the migration length of In adatom and enhance the dot density, which is believed to be the origin of the PL intensity enhancement. As can be seen in Fig.1(b) and (c), the QDs grown on the misoriented substrates have smaller PL linewidth and stronger PL intensity than those grown on the exact substrates. It indicates that the QDs grown on
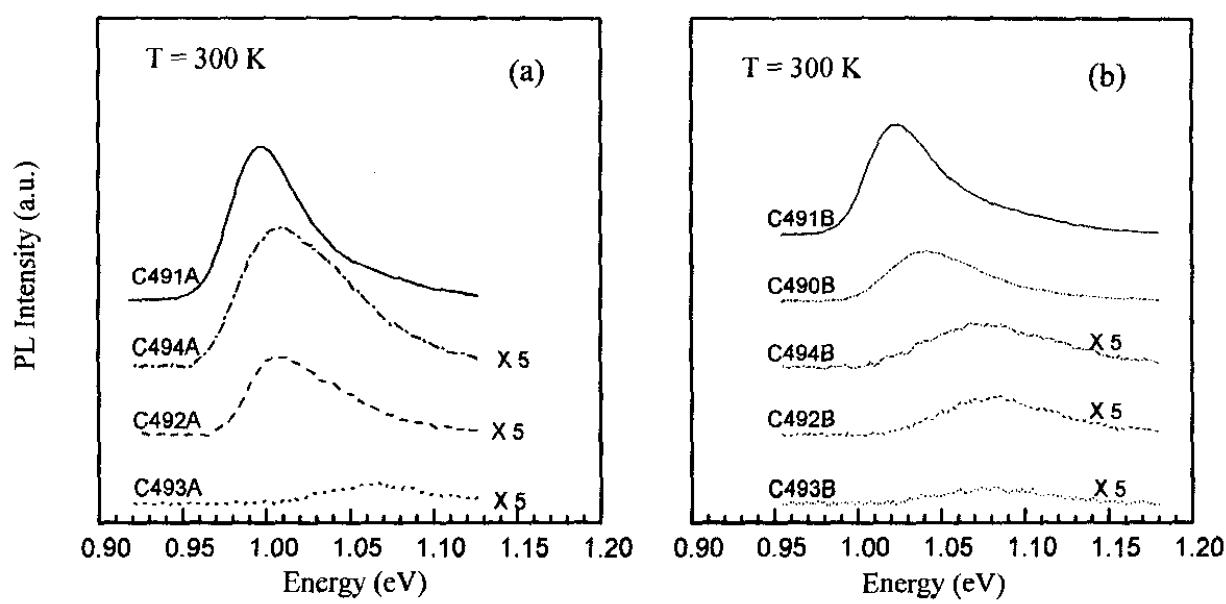

Fig. 3. The PL spectra at $300 \mathrm{~K}$ of the QDs grown on the exact (a) and vicinal (b) (100) GaAs substrates. 


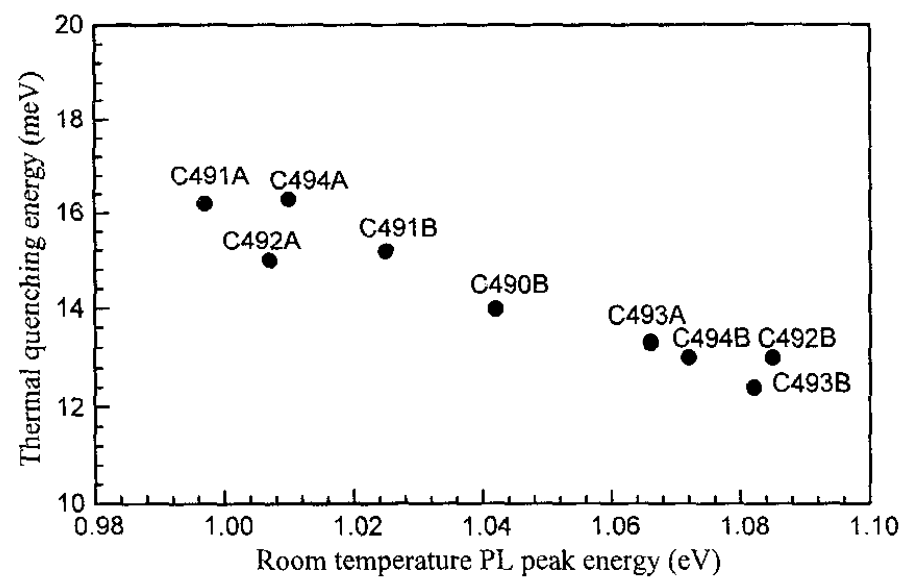

Fig. 4. Plots of the thermal quenching energy as a function of the room temperature (300 K) PL peak energy.

the misoriented substrates have better uniformity and higher dot density. Wavelength-integrated PL intensity as a function of thermal energy, $\mathrm{kT}$, for QDs grown on the exact and misoriented substrates are shown in Fig.2(a) and (b), respectively. As can be seen, QDs grown on the misoriented substrates have stronger PL intensities as temperature change from 8.5 to $120 \mathrm{~K}$. However, when temperature is higher than $120 \mathrm{~K}$, the PL intensities degrade faster than those of grown on the exact substrates. This phenomenon is especially noticeable for C492B, C493B and C494B. From these plots, the thermal quenching energy and the activation energy from exciton level to nonradiative level are determined and summarized in Table 1. The PL spectra at $300 \mathrm{~K}$ of the QDs grown on the exact and vicinal substrates are shown in Fig. 3(a) and (b), respectively. It is found the QDs grown at a V/III ratio of 2 showing the strongest PL intensity for both types of substrates, which is attributed to the largest dot size under this As-stabilized circumstance. The spectrum of C490A is not shown in the figure because its PL intensity is too weak to be available. Fig. 4 plots the thermal quenching energy as a function of the room temperature PL peak energy. It is found that the thermal quenching energy is increasing with the decreasing $300 \mathrm{~K} \mathrm{PL}$ peak energy, which indicates that the larger the dot size the better the optical property at $300 \mathrm{~K}$.

\section{Conclusions}

Self-organized InAs QDs grown on both (100) exact and misoriented $7^{\circ}$ toward (110) GaAs substrates by using GSMBE with different V/III ratio are characterized by PL measurements. The QDs grown on the misoriented substrates show narrower PL linewidths and stronger intensities at the same growth conditions, which implies that they are with good uniformity. Low temperature PL intensities increase with $\mathrm{AsH}_{3}$ flow rates for QDs grown on both two types of substrates at As-stabilized circumstance, which is attributed to the dot density increasing with the $\mathrm{As}_{2}$ pressure. The samples grown at a V/III ratio of 2 show the best PL characteristics at $300 \mathrm{~K}$. The study of thermal quenching energy reveals that the larger the dot size the better the PL intensity at $300 \mathrm{~K}$.

\section{References:}

[1] N. Kirstaedter et.al. 1994 Electron. Lett. 30 1416-7

[2] D. Bimberg et.al. 1996 Phys. Stat. Sol. (b) 194 159-73.

[3] D. I. Lubyshev et.al. 1996 J. Vac. Sci. Technol. B 14 2212 15

[4] A. Madhukar et.al. 1994 Appl. Phys. Lett. 64 2727-9

[5] Takayoshi Anan et.al. 1992 Appl. Phys. Lett. $603159-61$

[6] E. Tournie and K. H. Ploog 1994 J. Cryst. Growth $13597-112$ 